\title{
A More Thorough Transurethral Resection
}

\author{
Edward M. Messing* \\ University of Rochester Medical Center, Rochester, NY, USA
}

The standard initial treatment for urothelial cancer and other tumors of the bladder is a complete transurethral resection (TURBT). This is, of course, also a diagnostic and staging procedure and the information gained from both the endoscopic and histologic examinations have huge prognostic and management implications. But the hallmark of this procedure, particularly for non-muscle invading (NMI) urothelial cancers (UCs) is that all VISIBLE tumor is resected/ablated. With rare, although notable exceptions (e.g. too much tumor to safely resect at one sitting, recognized large bladder perforation early in the case, obvious deeply invasive cancer that is unresectable transurethrally, very diffuse suspected carcinoma-in-situ [CIS]) it is generally considered inappropriate to leave known tumor unsampled and untreated. The adverse clinical implications of doing this, including histological undersampling (and thus developing incorrect management plans) and requiring subsequent intravesical chemo or immunotherapeutic treatments to kill larger clumps of tumor than is optimal, can range from being unnecessarily morbid and costly for patients, to being catastrophic for them.

However, since even low grade (LG) NMI UC recurs frequently and rapidly [1], the possibility of incomplete resection/ablation with a TURBT is very real, in large part because of incomplete tumor visualization. That this is the case has been demonstrated in numerous randomized studies that have utilized the two most frequently tested methods for enhanced tumor visualization: Photodynamic diagnosis (PDD) and narrow band imaging [2-6].

\footnotetext{
${ }^{*}$ Correspondence to: Edward M. Messing, University of Rochester Medical Center, Rochester, NY, USA. Tel.: +1 585275 3345; E-mail: Edward.messing@urmc.rochester.edu.
}

PDD utilizes the fluorescence properties of a hematoporphyrin derivative, either 5-amminoleuvelenic acid (5-ALA) or hexylaminolevulinate (HAL). These are usually instilled intravesically, are preferentially taken up and retained by tumors, metabolized to protoporphyrin IX, and emit red fluorescence when excited by blue light. White light, of course, also contains blue light but the red fluorescence is difficulty to detect against the pinkish background of the bladder when viewed with the white light. Special equipment requiring cables and filters to generate the blue light and scopes that accentuate the red light are required. Additionally, the instilled hematoporphyrin, Cysview is the agent approved by the Food and Drug Administration (FDA) in the United States, must be instilled into the bladder and retained 60 minutes before the cystoscopic procedure for optimal visualization.

Narrow band imaging (NBI) filters white light into two discreet bands in the blue $(415 \mathrm{~nm})$ and green $(540 \mathrm{~nm})$ spectra, and accentuates the contrast seen primarily in blood vessels (mostly because of hemoglobin) in or just below the epithelium, compared to wide spectrum white light used for routine endoscopic visualization. It does not require instillation of an agent into the bladder.

Both modalities can be "turned on" by a switch on the scopes utilized, so that separate instruments do not have to be passed, and both have demonstrated enhanced tumor visualization compared to standard white light cystoscopy in numerous randomized prospective studies [2-7]. Two recent publications have tried to compare these two techniques.

In one, Lee, et al. [7] analyzed available randomized controlled trials of PDD (with 5-ALA or HAL) or NBI (all vs white light cystoscopy) and attempted to compare the performance of each in 
terms of tumor detection and recurrence rates. While initially 41 studies were screened, most were eliminated because they had inadequate or no follow-up for recurrence, or did not describe details about randomization, patient selection criteria or blinding of outcome information for detection or follow-up. The authors concluded that recurrence rates for tumors resected using HAL-based PDD were significantly higher than for 5-ALA-based PDD, but that neither form of PDD differed significantly from NBI in terms of recurrence. All were associated with significantly lower recurrence rates than white light cystoscopy. Intriguingly, the six 5-ALA studies analyzed were published in between 2001 and 2011, the five HAL studies between 2010 and 2013 and the four NBI studies between 2012 and 2014. This probably reflects in part when companies were trying to obtain regulatory approval; but it should be understood that many of the 5-ALA studies could not be completely compared with studies conducted with HAL because of changes that occurred with histologic grading classification of NMI urothelial cancers in 2004 [8]. Moreover, morbidities of treatments, particularly with the two PDD agents, were not discussed. Neither were false positive detections, a known problem with all three techniques. Finally, the study that gained approval for HAL in the United States, [4] was not included. While it may have been one of the studies eliminated from review, it's failure to be included is puzzling.

Importantly, while all methods were associated with a considerable reduction in recurrence rates compared to white light cystoscopy, none of the 10 studies which reported it, found significant improvement in reducing subsequent progression to stage T2. This is not only because progression rates were low in most studies, but also because most patients with tumors at high-risk for progression (e.g. high grade, stage T1, CIS) were treated with intravesical Bacillus Calmette Guerin (BCG) after TURBT. These factors had been brought up in an editorial by Herr [9] commenting on an article reporting higher detection rates of CIS using PDD than with white light cystoscopy [10].

In the second article, Drejer, et al. [11] reports a prospective study carried out in 4 institutions in Denmark and Norway, in which 171 patients with suspected NMI UC were each examined with white light, NBI and HAL PDD cystoscopy. While the authors stated that patients were at high risk for having CIS and in the 171 patients almost $80 \%$ had at least one biopsy performed because of suspicion of CIS, CIS only was diagnosed in 13 patients and flat dysplasia in another 10. Moreover, over 50\% of patients were undergoing TURBT/biopsy of their first lesion suspicious for bladder cancer. The authors reported sensitivities of $95.7 \%$ for both NBI and PDD, but only $65.2 \%$ for white light cystoscopy in diagnosing patients with UC. For each biopsy taken, the sensitivities for NBI and PDD were $72.7 \%$ and $78.2 \%$ for diagnosing CIS or flat dysplasia. White light cystoscopy had a $52.7 \%$ sensitivity on a per biopsy basis. This study did not report patient outcomes and it is presumed that most patients received BCG if they had high grade UC or CIS identified.

While this paper purported to compare the three modalities, since they were performed in the same order on every patient, it is very possible that urologists looked extremely intensely at the areas deemed suspicious looking on NBI when they examined the bladder using PDD. This would tend to make detection rates more similar with the two techniques than they otherwise might have been. Additionally, since the blue light component used in NBI shines light at the same wave length $(415 \mathrm{~nm})$ as in blue light cystoscopy, and since all patients had HAL instilled for one hour before any cystoscopic examination was performed, it is possible that the NBI appearance was more dramatic than it would normally (without HAL instillation) have been, because of accumulation of porphyrins in the microvasculature of tumors. Because of these factors, as well as the rather low overall incidence of CIS, (13\% rather than the 30\% assumed in the original power calculations), it is hard to be certain of the comparable sensitivities of NBI and PDD reported by the authors. While the possibility of the second modality biasing the findings of the third, could have been corrected by alternating the sequence of modalities used through randomization, [12] the possibility that HAL instillation improved the detection rate for NBI would not have been corrected. Only a prospective study of sufficient power in which patients who had NBI performed were randomized to receive a prior instillation of HAL or a placebo could correct for this bias.

That said, there is overwhelming evidence that both PDD and NBI perform significantly better than white light cystoscopy in detecting both papillary and flat NMI UC, and especially for LG papillary lesions where the confounding influence of intravesical immuno- or chemotherapy is eliminated. They reduce the rate of tumor recurrence. However, neither technique is used very often in our country. The expensive equipment not suitable for other uses, the high false positive rates of these modalities, 
the learning curve needed to become experienced with either, the inconvenience, expense, and discomfort (to patients) associated with pre-TURBT drug instillation (for PDD), and the additional time and complexity of the actual procedure with either modality, have all conspired to limit their use. Perhaps an easier-to-use method without employing long preoperative dwell times will become available - and allow us to do the most thorough TURBT we can.

\section{CONFLICT OF INTEREST}

Prof. Messing acts as an unpaid consultant for Imagen.

\section{REFERENCES}

[1] Messing EM, Kim K, Sharkey F, Schultz M, Parnes H, Kim D, et al. Randomized prospective phase III trial of difluoromethylornithine vs placebo in preventing recurrence of completely resected low risk superficial bladder cancer. J Urol 2006;176:500-4.

[2] Rink M, Babjuk M, Catto JW, et al. Hexyl aminolevulinateguided fluorescence cystoscopy in the diagnosis and follow-up of patients with non-muscle-inasive bladder cancer: A critical review of the current literature. European Urol 2013;64(4):624-38.

[3] Kausch I Sommerauer, Montorsi F, et al. Photodynamic diagnosis in non-muscle-invasive bladder cancer: A systemic review and cumulative analysis of prospective studies. Eur Urol 2008;53(6):1138-48.

[4] Grossman HB, Gomella L, Fradet Y, et al. A phase III multicenter comparison of hexaaminoluvelinate fluorescence cystoscopy and white light cystoscopy for the detection of superficial papillary lesions in patients with bladder cancer. J Urol 2007;178:62-67.

[5] Naselli A, Introini C, Timossi I, et al. A randomized prospective trial to assess the impact of transurethral resection in narrow band imaging modality on non-muscle-invasive bladder cancer recurrence. Eur Urol 2012;61(5):908-13.

[6] Li K, LinT, Fan X, Duan Y, Huang I. Diagnosis of narrowband-imaging in non-muscle-invasive bladder cancer: A systematic review and meta-analysis. International Journal of Urology: Official journal of the Japanese Urological Association 2013;20(6):602-9.

[7] Lee JY, Cho KS, Kang DH, et al. A network meta-analysis of therapeutic outcomes after new image technologyassisted transurethral resection for non-muscle invasive bladder cancer: 5-aminolaevulinic acid fluorescence vs hexylaminolevulinate fluorescence vs narrow band imaging. BMC Cancer 2015; 15:566.

[8] Eble JN, Sauter G, Epstein, JJ, et al. WHO Classification of Tumours: Tumours of the Urinary System and Male Genital Organs. IARC Press, Lyon 2004.

[9] Herr H. Editorial Comment. A comparison of hexylaminolevulinate fluorescence cystoscopy and white light cystoscopy for the detection of carcinoma in situ in patients with bladder cancer: A Phase III, Multicenter Study. J Urol 2007;178:73.

[10] Fradet Y Grossman HB, Gomella L, et al. A comparison of hexylaminolevulinate fluorescence cystoscopy and white light cystoscopy for the detection of carcinoma in situ in patients with bladder cancer: A Phase III, Multicenter Study. J Urol 2007;178:68-72.

[11] Drejer D, Beji S, Oezeke R, et al. Comparison of white light, photodynamic diagnosis and narrow-band imaging in detection of carcinoma in situ (CIS) or flat dysplasia at transurethral resection of the bladder: The DaBlaCa-8 study. Urology 2016, http://dx.doi.org/doi:10.1016/j.urology.20 16.11.032.

[12] Naya Y, Oishi M, Yamada Y, et al. Initial experience of combined use of photodynamic diagnosis and narrow band imaging for detection of flat urothelial lesion. Int J Clin Oncol 2015;20:593-7. 